\title{
An improvement within XFEM of the bonding between the enrichment area and the classical finite elements
}

\author{
Elie Chahine* - Patrick Laborde ${ }^{* *}$ — Yves Renard $* * *$ \\ * Laboratory for Nuclear Materials \\ Nuclear Energy and Safety Research Department \\ Paul Scherrer Institute OVGA/14 \\ CH-5232 Villigen PSI, Switzerland \\ elie.chahine@psi.ch \\ ** Institut de Mathématiques de Toulouse, UMR CNRS 5215 \\ Université Paul Sabatier (Université de Toulouse) \\ 118 route de Narbonne, 31062 Toulouse cedex 4, France \\ patrick.laborde@math.ups-tlse.fr \\ *** Université de Lyon, CNRS, INSA-Lyon, ICJ UMR5208 \\ LaMCoS UMR5259, F-69621, Villeurbanne, France \\ yves.renard@insa-lyon.fr
}

ABSTRACT. We are interested in XFEM strain calculations of a cracked elastic body. It is already known that with XFEM, the approximation quality is distorted by the layer of elements lying between the singular enrichment area and the rest of the mesh. In the following work, we replace this transition layer by a "mortar" type integral bonding condition at the interface between the two areas. We prove how the proposed approach enhance significantly the approximation.

RÉSUMÉ. On s'intéresse au calcul des déformations d'un corps élastique fissuré par la méthode des éléments finis étendue. On sait que la qualité de l'approximation est perturbée par la couche des éléments finis de transition entre la zone d'enrichissement autour du fond de fissure et le reste du maillage. On propose de remplacer la couche de transition par une condition intégrale, de type "raccord mortar», à l'interface entre ces deux zones. Nous montrons comment cette variante de XFEM permet d'améliorer sensiblement la précision.

KEYWORDS: crack, XFEM, non-conformal approximation.

MOTS-CLÉS : fissure, XFEM, approximation non conforme.

DOI:10.3166/EJCM.19.177-187 (C) 2010 Lavoisier, Paris 


\section{Introduction}

In this work, we are interested in numerical simulations of a cracked elastic isotropic linear body using a mesh of the non-cracked domain (independent of the crack path). The definition of the XFEM method on this mesh is done by "enriching" the classical finite element basis by functions that takes into account the a priori knowledge we have on the solution (Moës et al., 1999). For instance, the knowledge of the asymptotic expansion of the displacement field at the crack tip provides the "singular" enrichment that we apply to the nodes lying in an area surrounding the crack tip.

It is known that the approximation quality is distorted at the transition layer which is the layer of elements partially enriched, lying between the singular enrichment area and the rest of the domain (see (Chessa et al., 2003) in another context and (Laborde et al., 2005)). Moreover, in order to reduce the computational cost by reducing the number of additional degrees of freedom and to improve the conditioning, it is interesting to use a cut-off function that "globalizes" the enrichment over given surface (Chahine et al., 2005). Meanwhile, the transition between the singular enrichment and the classical elements remains a drawback. Therefore, the idea of this work is to have an XFEM variant that allows to bypass this difficulty by removing the transition layer. A more detailed presentation and analysis of this can be found in the $\mathrm{PhD}$ thesis (Chahine, 2008).

\section{A non-conformal approximation method}

Let us consider the planar cracked domain $\Omega, \Gamma_{C}$ denotes the crack. To simplify, $\Omega$ is assumed to be polygonal and $\Gamma_{C}$ a straight line. Les us denote by $\Omega_{1}$ and $\Omega_{2}$ a partition of the non-cracked domain $\bar{\Omega}$ such that the cracks tip $x^{*}$ belongs to $\Omega_{2}$ and the boundary $\partial \Omega_{2}$ of $\Omega_{2}$, is polygonal (Figure 1).

Let $\mathcal{T}_{h}$ be a triangulation of the non-cracked domain $\bar{\Omega}$ such that the interface $\partial \Omega_{2}$ between $\Omega_{1}$ and $\Omega_{2}$ coincides with sides of elements of $\mathcal{T}_{h}$, where h denotes the mesh parameter. The finite element mesh is then independent of the crack path. We define on $\mathcal{T}_{h}$ a $P_{1}$ finite element method whose scalar basis functions are denoted $\left\{\varphi_{i}\right\}_{i \in I}$. The shape function $\varphi_{i}$ corresponds to the node $x_{i}$. Let $I_{H}$ be the set of node indices "enriched" by the Heaviside type function:

$$
H(x)= \begin{cases}+1 & \text { if }\left(x-x^{*}\right) \cdot n \geq 0 \\ -1 & \text { elsewhere, }\end{cases}
$$

where $n$ is the normal vector to $\Gamma_{C}$. In other words, $I_{H}$ is the set of nodes whose corresponding shape functions have their support entirely cut by the crack (see (Moës et al., 1999)). Let also $I\left(\Omega_{k}\right)$ (resp. $\left.I_{H}\left(\Omega_{k}\right)\right)$ be the subset of indices $i \in I$ (resp. $\left.i \in I_{H}\right)$ such that $x_{i} \in \Omega_{k}, k \in\{1,2\}$. 


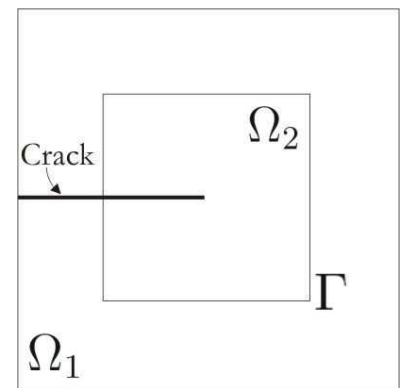

Figure 1. Partitioning of the cracked domain

Let $\mathcal{V}_{1}^{h}$ be the space of the $P_{1}$ vector finite elements functions associated to the induced triangulation on $\Omega_{1}$ and enriched by the jump function $H$ :

$$
\mathcal{V}_{1}^{h}=\left\{v_{1}^{h}: v_{1}^{h}=\sum_{i \in I\left(\Omega_{1}\right)} a_{i} \varphi_{i}+\sum_{i \in I_{H}\left(\Omega_{1}\right)} b_{i} H \varphi_{i} ; a_{i}, b_{i} \in \mathbb{R}^{2}\right\} .
$$

Moreover, the space $\mathcal{V}_{2}^{h}$ is the space of the vector finite element functions on $\Omega_{2}$ enriched, not only by the jump function $H$, but also by the "singular" functions at the crack tip:

$$
\mathcal{V}_{2}^{h}=\left\{v_{2}^{h}: v_{2}^{h}=\sum_{i \in I\left(\Omega_{2}\right)} a_{i} \varphi_{i}+\sum_{i \in I_{H}\left(\Omega_{2}\right)} b_{i} H \varphi_{i}+\sum_{j=1}^{4} c_{j} F_{j} ; a_{i}, b_{i}, c_{j} \in \mathbb{R}^{2}\right\},
$$

where the enrichment functions $F_{j}$ are give by polar coordinates by (see (Moës et al., 1999)):

$$
\left\{F_{j}(x)\right\}_{1 \leq j \leq 4}=\left\{\sqrt{r} \sin \frac{\theta}{2}, \sqrt{r} \cos \frac{\theta}{2}, \sqrt{r} \sin \frac{\theta}{2} \sin \theta, \sqrt{r} \cos \frac{\theta}{2} \sin \theta\right\} .
$$

The discrete displacement field defined in the cracked domain will belong to $\mathcal{V}^{h}$, the space of functions $v^{h}$ defined on $\Omega$ such that $(k \in\{1,2\})$ :

$$
v^{h}=v_{k}^{h} \quad \text { in } \Omega_{k}, \quad \text { where } v_{k}^{h} \in \mathcal{V}_{k}^{h} .
$$

The global approximation space $\mathcal{V}^{h}$ can be identified to the product space $\mathcal{V}_{1}^{h} \times \mathcal{V}_{2}^{h}$.

The subdomain $\Omega_{2}$ will be the enrichment surface where the singular crack tip functions will be applied. Let us note that the introduced singular functions in (see the definition of $\mathcal{V}_{2}^{h}$ ) are "globalized" over $\Omega_{2}$ and so, they add only 8 degrees of freedom to the whole finite element method

Naturally, this approximation method defined from the discrete displacement fields is a non-conformal one, the global displacement field in $\mathcal{V}^{h}$ is not continuous through 
the interface between $\Omega_{1}$ and $\Omega_{2}$. In the following section, we define a bonding condition in order to retrieve a continuity property to the solution through the interface.

\section{An integral matching condition}

The jump of the displacement field $u$ at the interface can be written:

$$
[u]=\left.\left(u_{2}-u_{1}\right)\right|_{\Gamma}
$$

where $\Gamma=\partial \Omega_{2} \cap \Omega$ denotes the interface "cut" by the crack and $u_{k}$ the restriction of $u$ on $\Omega_{k}$. Let then

$$
b(u, \mu)=-\int_{\Gamma} \mu \cdot[u] d \Gamma
$$

for all the multipliers $\mu$.

In order to have a continuity condition at the interface $\Gamma$ in the discontinuous approximation space $\mathcal{V}^{h}$, we define the following discrete multipliers. The triangulation $\mathcal{T}_{h}$ introduced in Section 2 defines a subdivision $\mathcal{S}_{h}$ of the "non-cut" interface $\bar{\Gamma}=\partial \Omega_{2}$ into elementary segments. The space $\mathcal{W}^{h}$ of discrete multipliers can be written

$$
\mathcal{W}^{h}=\left\{\mu^{h} \in C^{0}(\bar{\Gamma})^{2}:\left.\mu_{i}^{h}\right|_{S} \in P_{1}, \forall S \in \mathcal{S}_{h}, i \in\{1,2\}\right\} .
$$

Let un note that the latter definition does not take into account the discontinuity through the crack sides.

We consider the following hybrid formulation that correspond to a discrete form of the linear elasticity problem over the cracked domain $\Omega$ (see (Brezzi et al., 1991))

$$
\text { Find } u^{h} \in \mathcal{V}^{h}=\mathcal{V}_{1}^{h} \times \mathcal{V}_{2}^{h}, \lambda^{h} \in \mathcal{W}^{h} \text { such that }
$$

$$
\begin{aligned}
& a\left(u^{h}, v^{h}\right)+b\left(v^{h}, \lambda^{h}\right)=L\left(v^{h}\right) \text { for all } v^{h} \in \mathcal{V}^{h}, \\
& b\left(u^{h}, \mu^{h}\right)=0 \text { for all } \mu^{h} \in \mathcal{W}^{h} .
\end{aligned}
$$

the bilinear form $a$ is defined by:

$$
a(u, v)=\sum_{k=1}^{2} a_{k}\left(u_{k}, v_{k}\right)=\sum_{k=1}^{2} \int_{\Omega_{k}} D \varepsilon\left(u_{k}\right): \varepsilon\left(v_{k}\right) d x,
$$

where $D$ is the material stiffness operator. Moreover,

$$
L(v)=\sum_{k=1}^{2} \int_{\Omega_{k}} g \cdot v_{k} d x+\int_{\Gamma_{N}} f \cdot v_{1} d \Gamma,
$$


where $f$ and $g$ denotes respectively the given loads applied respectively on the part $\Gamma_{F}$ of the boundary and inside the body $\Omega$. To simplify, we do not take into account the contact of the crack sides during the deformation.

The approximation space $\mathcal{V}^{h}$ cannot be identified to a subspace of the elasticity classical Sobolev space $H^{1}(\Omega)^{2}$. In the introduced non-conformal method, the continuity of the discrete displacement field in $\mathcal{V}^{h}$ through the interface $\Gamma$ is defined only in a weak discrete sense; this is the integral matching condition [9b]. The pointwise matching condition:

$$
\left[u_{h}\right]=0 \text { on every node of } \Gamma,
$$

provides another type of approximation of the continuity condition at the transition.

\section{Convergence rate}

\subsection{Problem position}

The aim of this section is to obtain the convergence error that satisfies the solution of the discrete problem [9] when the mesh parameter $h$ tends to zero. In what follows, the variational formulation of the continuous problem is given.

Let $\mathcal{V}$ the space of the discontinuous displacements across the interface between $\Omega_{1}$ and $\Omega_{2}$ :

$$
\left.\mathcal{V}=\left\{v \in L^{2}(\Omega)\right)^{2}: v_{k}=\left.v\right|_{\Omega_{k}} \in H^{1}\left(\Omega_{k}\right)^{2}, v_{1}=0 \text { on } \Gamma_{D}\right\} .
$$

The space $\mathcal{V}$ can be identified to the space $\mathcal{V}_{1} \times \mathcal{V}_{2}$ equipped with the canonical norm $\|\cdot\|_{\mathcal{V}}$ of the product space $H^{1}\left(\Omega_{1}\right)^{2} \times H^{1}\left(\Omega_{2}\right)^{2}$.

The continuity condition $[u]=0$ across $\Gamma$ of the displacement field $u$ can be written in an integral form as follows

$$
b(u, \mu)=0 \quad \text { for all } \mu \text { in } \mathcal{W},
$$

where the multiplier space $\mathcal{W}$ is the dual space $\left(H^{1 / 2}(\Gamma)^{2}\right)^{\prime}=H_{00}^{-1 / 2}(\Gamma)^{2}$ equipped with its canonical norm denoted $\|\cdot\|_{-1 / 2, \Gamma}$.

Then, the continuous linear elasticity problem over the cracked diomain can be written

Find $u \in \mathcal{V}=\mathcal{V}_{1} \times \mathcal{V}_{2}, \lambda \in \mathcal{W}$ such that

$$
\begin{aligned}
& a(u, v)+b(v, \lambda)=L(v) \text { for all } v \in \mathcal{V}, \\
& b(u, \mu)=0 \text { for all } \mu \in \mathcal{W} .
\end{aligned}
$$

The following studies the convergence error $\left\|u-u_{h}\right\|_{\mathcal{V}}$ between the solution to the latter problem and the one of the discrete problem [9] when $h$ tends to zero. 


\subsection{An error estimate}

The convergence rate of the proposed approximation method goes through several steps. The detailed proofs can be found in (Chahine et al., To appear in Applied Numerical Mathematics).

First, one should prove that that the discrete spaces $\mathcal{V}^{h}$ and $\mathcal{W}^{h}$ of the discrete displacements and multipliers resp. satisfy a uniform compatibility condition:

$$
\inf _{\mu^{h} \in \mathcal{W}^{h}{ }_{v^{h} \in \mathcal{V}^{h}}} \sup _{\left\|v^{h}\right\|_{\mathcal{V}}\left\|\mu^{h}\right\|_{-1 / 2, \Gamma}} \geq \beta,
$$

where $\beta>0$ is a constant independent of $h$.

Another difficulty exists because of the non-conformity character of the approximation method: the coerciveness of the "broken" bilinear form $a$ is not satisfied in the discrete displacements space $\mathcal{V}^{h}$. Meanwhile, one can prove a coerciveness property in the subspace

$$
V_{0}=\left\{w \in \mathcal{V}: \int_{\Gamma} \mu_{i} \cdot w d \Gamma=0, i \in\{1,2,3\}\right\},
$$

where

$$
\mu_{1}(x)=(1,0), \mu_{2}(x)=(0,1), \mu_{3}(x)=\left(\alpha_{1}, x_{1}+\alpha_{2}\right), \quad \forall x \in \Gamma,
$$

with

$$
\alpha_{1}=\gamma_{1} / \gamma, \alpha_{2}=\gamma_{2} / \gamma
$$

and

$$
\gamma=\int_{\Gamma} 1 d \Gamma, \gamma_{1}=\int_{\Gamma} x_{1} d \Gamma, \gamma_{2}=\int_{\Gamma} x_{2} d \Gamma, \gamma_{3}=\int_{\Gamma}\left(x_{1}^{2}+x_{2}^{2}\right) d \Gamma
$$

Thanks to this latter coerciveness property together with the inf-sup condition [16], the following abstract error estimate can be obtained between the solutions $u$ and $u_{h}$ to Problems [15] and [9] resp.:

$$
\left\|u-u^{h}\right\|_{\mathcal{V}}^{2}+\left\|\lambda-\lambda^{h}\right\|_{-1 / 2, \Gamma}^{2} \leq C\left\{\inf _{v^{h} \in \mathcal{V}^{h}}\left\|u-v^{h}\right\|_{\mathcal{V}}^{2}+\inf _{\mu^{h} \in \mathcal{W}^{h}}\left\|\lambda-\mu^{h}\right\|_{-1 / 2, \Gamma}^{2}\right\}
$$

where $C$ denotes a constant independent of $h$.

Then, let us point out another essential point of the mathematical analysis of the integral matching method, which is the approximation of a multiplier $\mu$ defined on the 


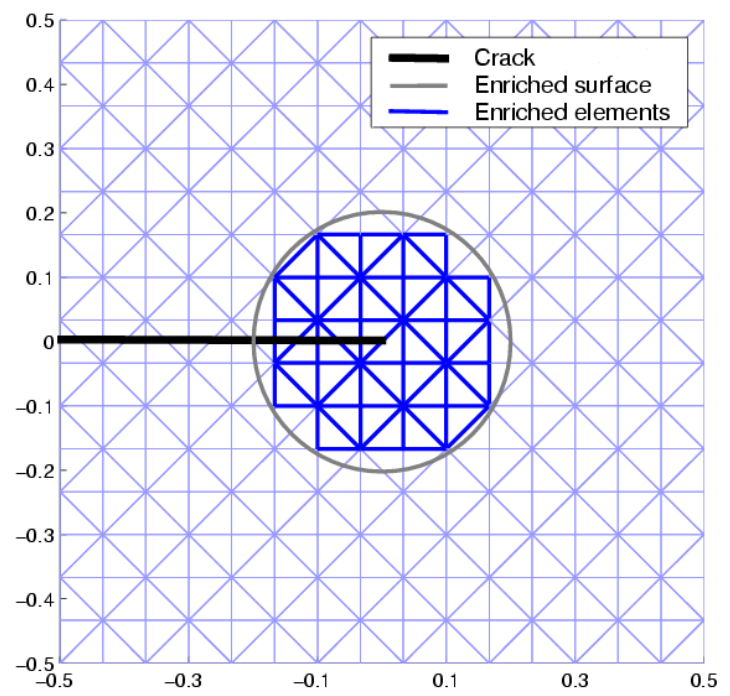

Figure 2. Finite element mesh of the non-cracked domain and singular enrichment area

"cracked" interface $\Gamma$ by the discrete multipliers $\mu_{h}$. If $\mu \in H^{1 / 2}(\Gamma)^{2}$, one can prove that

$$
\inf _{\mu^{h} \in \mathcal{W}^{h}}\left\|\mu-\mu^{h}\right\|_{-1 / 2, \Gamma} \leq C h\|\mu\|_{1 / 2, \Gamma}
$$

where $\|\cdot\|_{1 / 2, \Gamma}$ denotes the $H^{1 / 2}(\Gamma)^{2}$-norm. Note that the mathematical study of the approximation of a displacement field in $\mathcal{V}$ by displacements in the finite element space $\mathcal{V}^{h}$ is based on an elementary analysis achieved in (Chahine et al., 2005).

We assume that the solution $(u, \lambda)$ to problem [9] is sufficiently regular. More precisely, the exact displacement $u$ can be written $u=u_{r}+u_{s}$ where $u_{s}$ is the asymptotic displacement at the crack tip defined using the stress intensity factors. Moreover, the regular part $u_{r}$ satisfies

$$
u_{r}=u-u_{s} \in H^{2+\varepsilon}\left(\Omega, \mathbb{R}^{2}\right),
$$

for all $\varepsilon>0$ and that the exact multiplier $\lambda \in H^{1 / 2}(\Gamma)^{2}$.

Then the solution $\left(u^{h}, \lambda^{h}\right)$ to Problem [9] satisfies

$$
\left\|u-u^{h}\right\|_{\mathcal{V}}+\left\|\lambda-\lambda^{h}\right\|_{-1 / 2, \Gamma} \leq C h,
$$

where $C$ is a constant independent of $h$.

In other words, the approximation error is optimal: it has the same order of a classical conformal finite element method defined on a non-cracked domain. 

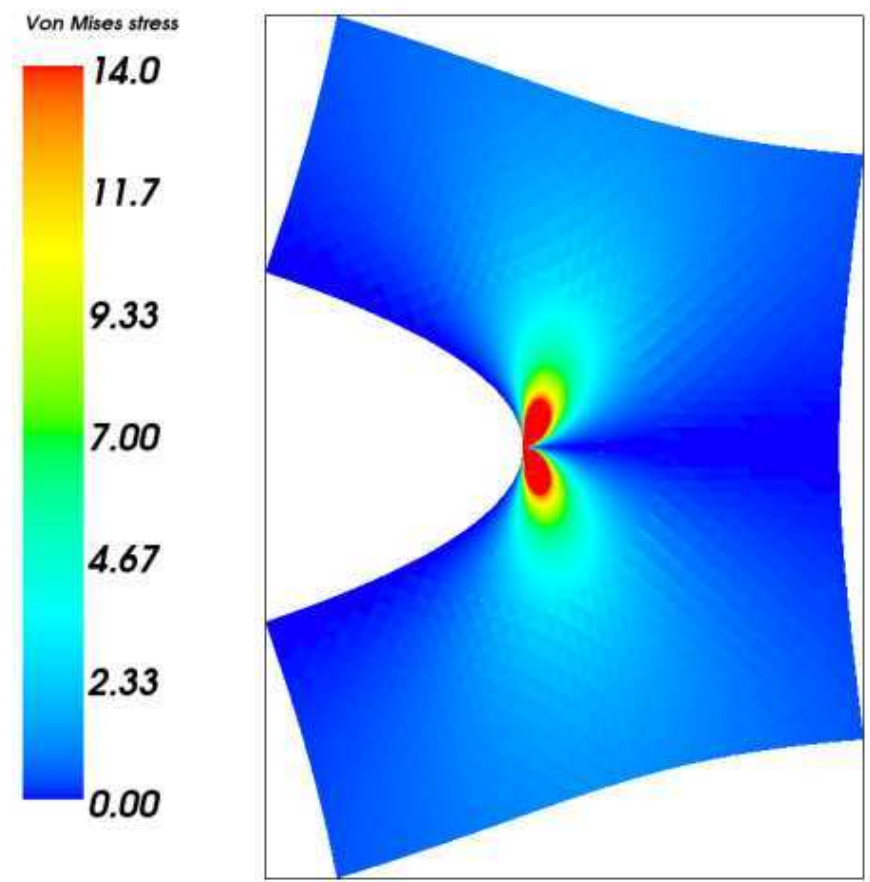

Figure 3. Von Mises stress for a mode I problem using $P_{1}$ elements with the integral matching XFEM

\section{Numerical simulations}

The following simulations where performed on the crackd domain:

$$
\Omega=]-0.5 ; 0.5[\times]-0.5 ; 0.5\left[\backslash \Gamma_{C},\right.
$$

where $\Gamma_{C}$ denotes the crack given by:

$$
\Gamma_{C}=[-0.5 ; 0] \times\{0\} .
$$

The boundary conditions non-homogeneous Dirichlet ones given by the exact opening mode $u_{I}$ (see (Lemaitre et al., 1994)).

A $P_{1}$ finite element method is defined on a structured mesh of the non-cracked domain $\bar{\Omega}$ (see Figure 2). The singular enrichment area (Section 2) is chosen as the set of elements contained in the ball $B\left(x^{*}, 0.2\right)$, where $x^{*}$ denotes the crack tip. The XFEM method was implemented using GETFEM++, a C++ object oriented finite element library developed in our team (see (Renard et al., http://home.gna.org/getfem)). 


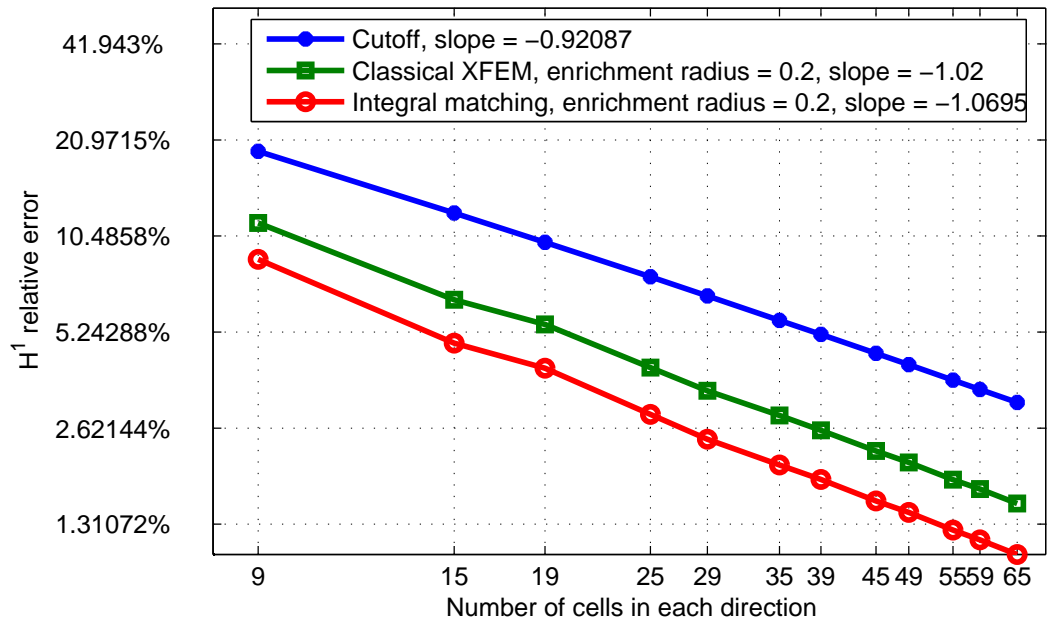

Figure 4. $H^{1}$-error with respect to the number of cells in each direction for a mode I problem with enriched $P_{1}$ elements (logarithmic scales)

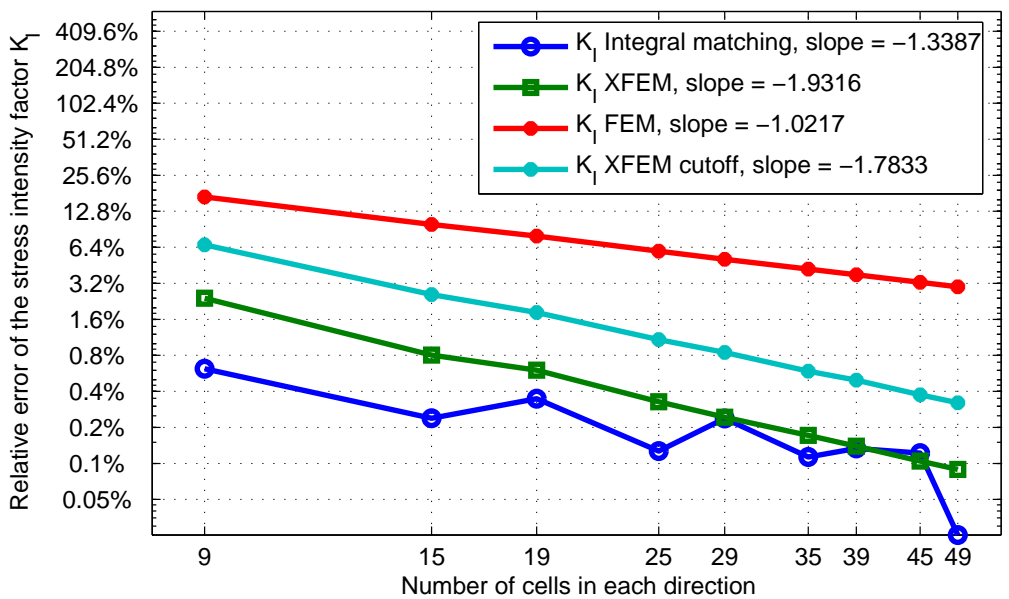

Figure 5. Relative error with respect to the number of cells in each direction with enriched $P_{1}$ elements (logarithmic scales) 
Figure 3 show the level-set lines of the Von Mises stress over the deformed structure. Around the crack tip and at the transition layer neighboring the boundary of the singular enrichment area, one observes that the filed remains very regular comparing to the conformal XFEM method (see (Laborde et al., 2005)).

Figure 4 compare the error convergence curves, when the mesh parameter $h$ goes to zero, obtained for an XFEM conformal method with surface enrichment (see (Béchet et al., 2005), (Laborde et al., 2005)), for the cut-off XFEM (see (Chahine et al., 2005)) and for the integral matching XFEM approach introduced in this paper. These errors are computed with the energy norm and drawn as function of the number $N=1 / h$ of the elements in each direction.

Other simulations showed that the numerical results are similar when dealing with a non-structured mesh or with a mixed mode fracturing problem.

Finally, Figure 5 illustrates the values of the first stress intensity factor $K_{I}$. They are computed using the integral $J$ method (see (Moës et al., 1999) for the contour integral computation). The defined exact solution is a linear combination between the two modes $u_{I}$ and $u_{I I}$ and a given regular solution on the non-cracked domain: $P(x)+$ $3 u_{I}+5 u_{I I}$. The error convergence curves of the classical finite element method (FEM), the cut-off XFEM and the surface enrichment XFEM are smooth, but overestimate the exact value of the SIF $K_{I}$. The integral matching XFEM oscillate around this value but gets closer to it than the other approaches. For further numerical results, see (Chahine et al., To appear in Applied Numerical Mathematics).

To conclude, we showed how the integral matching XFEM increases the numerical precision by improving the approximation quality around the crack tip and at the transition layer between the singular enrichment area and the rest of the domain. This is validated in the mathematical study as well as in the illustrated numerical simulations.

\section{References}

Béchet E., Minnebo H., Moës N., Burgardt B., « Improved implementation and robustness study of the X-FEM for stress analysis around cracks », Int. J. Numer. Meth. Engng., vol. 64, p. 1033-1056, 2005.

Brezzi F., Fortin. M., Mixed and Hybrid Finite Element Methods, Springer, 1991.

Chahine E., Etude mathématique et numérique de métodes d'éléments finis étendues pour le calcul en domaines fissurés, Phd thesis, INSA Toulouse, Université de Toulouse, 2008.

Chahine E., Laborde P., Renard Y., « Crack tip enrichment in the XFEM method using a cut-off function », Int. J. Numer. Meth. Engng., vol. 64, p. 354-381, 2005.

Chahine E., Laborde P., Renard Y., « Integral matching XFEM : a non-conformal eXtended Finite Element approach », To appear in Applied Numerical Mathematics.

Chessa J., Wang H., Belytschko T., « On the construction of blending elements for local partition of unity enriched finite elements », Int. J. Numer. Meth. Engng., vol. 57, p. 1015-1038, 2003. 
Laborde P., Renard Y., Pommier J., Salaün M., « High order extended finite element method for cracked domains », Int. J. Numer. Meth. Engng., vol. 64, p. 354-381, 2005.

Lemaitre J., Chaboche J., Mechanics of Solid Materials, Cambridge University Press, Cambridge, 1994.

Moës N., Dolbow J., Belytschko T., « A finite element method for crack growth without remeshing », Int. J. Numer. Meth. Engng., vol. 46, p. 131-150, 1999.

Renard Y., Pommier J., Getfem++. An open source generic C++ library for finite element methods, Technical report, http://home.gna.org/getfem. 
